\title{
Estudio sobre el patrimonio de la ciudad de Lorca en educación primaria
}

\section{Research about heritage in the city of Lorca in primary education}

\author{
Silvia Martínez de Miguel López \\ Universidad de Murcia (España) \\ silviana@um.es \\ Juan Antonio Salmerón Aroca \\ Universidad de Murcia (España) \\ jasa2@um.es \\ Maria Luz Serrano Ruiz \\ Universidad de Murcia (España) \\ serrano@um.es \\ Fecha de recepción: 15/02/2019 \\ Fecha de aceptación: 24/07/2019
}

\section{Resumen}

El objetivo de este trabajo es analizar el nivel de conocimientos, que posee el alumnado de educación primaria de Lorca (Murcia), acerca de los bienes culturales de la ciudad después del seísmo que asoló a la ciudad en 2011. En el marco de un diseño de investigación no experimental, de tipo exploratorio, se empleó un cuestionario creado ad hoc, que fue aplicado a 156 escolares de educación primaria. Los resultados del estudio muestran una evidente capacidad de mejora respecto al nivel de conocimiento y grado de frecuentación del alumnado al patrimonio histórico artístico. Se concluye que, a pesar de más de un lustro de rehabilitación del patrimonio lorquino, sin embargo, el conocimiento por parte del alumnado es todavía escaso frente a sus posibilidades pedagógicas, siendo necesario promover e incrementar su frecuentación, difusión, divulgación y conocimiento desde la escuela.

Palabras clave: Educación primaria; Educación patrimonial; Patrimonio cultural; Museo; Lorca (Murcia, España) 
Estudio sobre el patrimonio de la ciudad de Lorca... - S. Martínez de Miguel y otros

\begin{abstract}
The objective of this work is to analyze the level of knowledge of primary education students about the cultural and museum heritage, after having suffered the earthquake that devastated the city on 2011. A non-experimental research design, of an exploratory type, was used an ad hoc questionnaire, which was applied to 156 primary school students. The results of the study show an evident improvement in the level of knowledge and degree of attendance of the local cultural heritage. It is concluded that, despite after more than a few years of rehabilitation of the lorquino heritage, however, the knowledge of the students is still low compared to their pedagogical possibilities, being necessary to promote and to increase their frequency, dissemination, dissemination and knowledge from school.
\end{abstract}

Keywords: Primary education; Heritage education; Cultural heritage; Museum; Lorca (Murcia, Spain)

\title{
1. INTRODUCGIÓN
}

En un intento de aproximación a las prácticas docentes que favorecen la enseñanza del patrimonio, y siguiendo las recomendaciones de Calaf, San Fabián y Gutiérrez (2017), acerca de la necesidad de nuevos enfoque evaluativos en la intervención educativa en museos, se ha considerado importante analizar los conocimientos que los alumnos poseen acerca del patrimonio y los museos de su entorno, así como las visitas que realizan para identificar posibles áreas de actuación y mejora que permitan ofrecerles experiencias satisfactorias. Se plantea su realización en relación con el alumnado de sexto de educación primaria de la ciudad de Lorca, ya que de esta forma, los datos obtenidos indicarían las características de este conocimiento al terminar esta etapa educativa, para así, poder desarrollar estrategias de mejora durante toda la educación primaria. Es necesario hacer mención al ámbito geográfico donde se ha desarrollado este estudio. Lorca, cuyo centro urbano fue declarado conjunto histórico-artístico en 1964, es conocida como la ciudad barroca por el importante legado barroco de su centro histórico, uno de los de mayor proyección de la región de Murcia, y por los hechos históricos que han ido conformando la Lorca actual desde el paleolítico hasta la actualidad. Los yacimientos arqueológicos, su castillo, convertidos en un espacio temático. Además de ello, el parque arqueológico y sinagoga, que guarda en su interior del recinto amurallado del Castillo uno de los descubrimientos más interesantes realizados durante las excavaciones arqueológicas que aún hoy continúan: los restos de una judería de los siglos XIV-XV. Se trata de un conjunto de casas dispuestas en varias terrazas con un trazado de calles que las conecta formando un extenso barrio que tiene como elemento principal una Sinagoga en un estado de conservación muy bueno; las numerosas iglesias y conventos de diferentes épocas y estilos (s. XIII al s. XIV) los palacios y casas señoriales del barroco, como el Palacio de Guevara, la fortaleza militar -que reestructura la alcazaba medieval en un inexpugnable recinto- son algunos de los ejemplos del patrimonio artístico de la ciudad, también conocida como "Ciudad del Sol". Sin olvidar los desfiles bíblicopasionales de la Semana Santa lorquina declarada de Interés Turístico Internacional, 
Revista de Humanidades, 38 (2019). p.11-36. ISSN 1130-5029

con el fervor de blancos y azules, y sus valiosos bordados en seda y oro, algunos de ellos declarados Bienes de Interés Cultural. La ciudad de la frontera, de época musulmana y cristiana que hacen de la urbe uno de los centros históricos más importantes del levante español. Sin embargo, recientemente la ciudad de Lorca fue asediada por los terremotos del 11 de mayo de 2011, que causaron numerosos daños en el patrimonio monumental de la ciudad viéndose afectados edificios religiosos y construcciones, además de otros valiosos inmuebles. El proceso de reconstrucción de los mismos es lento, complejo y costoso, por el importante daño producido y ha sacado a la luz la necesidad de la percepción en la ciudadanía del valor que supone el patrimonio cultural para la ciudad. Desde estas consideraciones, se encuentra relevante saber cuál es el conocimiento y percepción en edades en las que se debe iniciar la sensibilización al respecto como señas de identidad cultural (Concejalía de Turismo, Educación y Universidad de Lorca, 2018).

\section{MARCO TEÓRICO}

La Organización de las Naciones Unidas para la Educación, la Ciencia y la Cultura (UNESCO, 2011), reconoce los bienes culturales en su sentido amplio incluyendo, tanto las obras arquitectónicas, esculturas o pinturas, las construcciones, y los lugares, que tienen un valor universal excepcional desde el punto de vista de la historia, el arte o la ciencia, así como también incluye tradiciones o expresiones de vida heredadas de nuestros antepasados y que se han ido transmitiendo a los descendientes, como las tradiciones orales, artes escénicas, prácticas sociales, rituales, eventos festivos, conocimientos y prácticas relativas a la naturaleza y al universo del conocimiento y de las habilidades, para producir artesanía tradicional. Monteagudo (2014), confirma así, que con ello se aleja definitivamente del concepto de patrimonio como visión materialista, que rinde culto al objeto, y se consolida una visión amplia y plural del mismo, valorándose todas aquellas entidades materiales e inmateriales de las distintas culturas, sin establecer límites temporales ni artísticos.

En este sentido el Ministerio de Educación del Gobierno de España, en consonancia con las directrices de las políticas emanadas del Consejo de Europa en el ámbito educativo en materia de patrimonio cultural (Jagielska-Burduk \& Stec, 2019), y ante la necesidad de coordinar las diferentes administraciones autonómicas y estatales para responder al enorme volumen de patrimonio que poseen, creó el Plan Nacional de Educación y Patrimonio (Ballesteros, Domingo, Fontal y Cirujano, 2013). Su propósito es elaborar desde el punto de vista psicopedagógico y didáctico, la normativa educativa en cuanto a los contenidos patrimoniales, que se deben incluir en los diferentes currículos, y junto al Observatorio de Educación Patrimonial, se centra en el inventario, análisis y evaluación de programas educativos, que en la actualidad ha devenido como el organismo estatal referente en el ámbito de la educación patrimonial (Fontal, Marín-Cepeda, García-Ceballos, Martínez y Sánchez, 2018) 
Estudio sobre el patrimonio de la ciudad de Lorca... - S. Martínez de Miguel y otros

Así, podemos decir que la educación patrimonial se adscribe fundamentalmente al potencial de este patrimonio como recurso didáctico, y como fuente básica del conocimiento social, tanto en ámbitos formales como no formales, inclusive en la educación superior (Fontal, 2003; Fontal y Marín, 2018). Castellón y Martínez (2001, p. 71), afirman al respecto: "sólo mediante el conocimiento podremos llegar primero a apreciar, luego a respetar y proteger y por último a disfrutar de los bienes culturales que constituyen la herencia colectiva de nuestro pueblo". Asimismo, Cuenca y Martín (2015), señalan que la finalidad de la educación patrimonial persigue la construcción de una ciudadanía socioculturalmente comprometida con los valores identitarios, el respeto intercultural y el cambio social. Para alcanzar este objetivo, autores como Fontal (2013), y estudios como los de Ibáñez, Fontal y Cuenca (2015), proponen partir del análisis, valoración y reconocimiento de los elementos del patrimonio cultural próximo al ciudadano. Si bien como afirman Fontal e Ibáñez (2015), todas estas medidas desarrolladas se pueden considerar acciones claves para evaluar el estado de la Educación Patrimonial en España, no obstante, se vislumbra la necesidad de poder trasladar estos conocimientos al currículum escolar. En este sentido Cuenca, Estepa y Martín (2011), afirman que parece predominar un enfoque unidisciplinar y tradicionalista, debido entre otros factores, a que las menciones al patrimonio en el currículum educativo español son limitadas y parciales tanto en la Ley Orgánica 2/2006, de 3 de mayo, de Educación (LOE), como en la Ley Orgánica 8/2013, de 9 de diciembre, para la Mejora de la Calidad Educativa (LOMCE). Pese a este tratamiento curricular, la enseñanza del patrimonio histórico artístico es un campo disciplinar lleno de posibilidades. Como señala González Monfort (2011), el uso del patrimonio histórico artístico contribuye a la formación de ciudadanos activos y críticos ayudando a desarrollar su capacidad para formular preguntas, investigar, contrastar informaciones, establecer relaciones de causalidad, y posicionarse de manera individual ante los problemas sociales asumiendo compromisos con el fin de lograr sociedades más justas basadas en el diálogo y el respeto a la diversidad cultural. Además, de acuerdo con Pinto (2013), el tiempo histórico es más comprensible para el alumnado cuando se desarrollan experiencias educativas en las que se les pone en contacto con fuentes históricas diversas. En esta misma línea de recreación histórica, e incluso identitaria, destaca como ejemplo aplicado, el estudio de Méndez(2017) sobre la necesidad de la educación patrimonial en el aprendizaje de asignaturas curriculares, que ayudan a profundizar en la interrelación de los bienes patrimoniales y la ciudadanía para la conformación de: "identidad, propiedad, pertenencia y emoción" (Fontal y Marín, 2018, p. 484)

Por tanto, puede afirmarse que el patrimonio histórico artístico puede ser utilizado en las aulas como un recurso educativo a partir del cual potenciar la construcción de una identidad ciudadana responsable y el desarrollo de un pensamiento social crítico, planteando alternativas a los grandes problemas de las sociedades actuales desde edades bien tempranas (Latorre y Mérida, 2019). Para lo cual distintos autores (Fontal, Ibáñez, Martínez, Rivero, 2017), coinciden en la necesidad de una 
Revista de Humanidades, 38 (2019). p.11-36. ISSN 1130-5029

formación específica para los futuros maestros durante la formación universitaria; o en palabras de Fontal (2003, p. 289): “conocer para comprender, comprender para respetar, respetar para valorar, valorar para cuidar, cuidar para disfrutar, disfrutar para transmitir, transmitir para conocer", con el fin de sensibilizar y lograr un aprendizaje significativo en relación con la educación patrimonial (en cualquiera de sus ámbitos, formal, no formal o informal). En este sentido, López Cruz (2014) desarrolla una propuesta de educación patrimonial para la enseñanza de las Ciencias Sociales que, en relación con el patrimonio y la identidad, apuesta por reconocer que el patrimonio lo forman los elementos simbólicos-identitarios, tanto de la cultura propia como la ajena, siendo necesario mostrar las peculiaridades patrimoniales de cada comunidad para favorecer el respeto y la conexión intercultural. Para ello, añaden Ott y Pozzi (2011), que una utilización adecuada de las TIC en el campo de la educación patrimonial abre una serie de nuevos escenarios, donde los límites de tiempo y espacio parecen disminuir o casi desaparecer. Sin embargo, algunas de las situaciones de enseñanza más significativas para el alumnado pueden llevarse a cabo fuera del aula, en sitios históricos, museos, etc., pudiendo realizar el profesorado una gran variedad de prácticas que, a través del contacto directo con el patrimonio local, promuevan sentimientos de pertenencia e involucren a los jóvenes en acciones presentes y futuras, tal y como recogen Molina y Pinto (2015). Las actividades didácticas en torno a los bienes culturales, sobre todo las que tienen carácter de salida escolar como las visitas a los museos, tienen además un fuerte componente motivacional para los estudiantes y contribuyen al desarrollo de la creatividad, el pensamiento divergente y el espíritu crítico, siendo necesario para ello la recíproca colaboración entre escuela y museo (Escribano y Molina, 2015b). Sin embargo, para superar su carácter meramente recreativo es necesaria una correcta relación entre la institución museística y la escuela (García y Gutierrez, 2018; Piedra, 2018). Así, resulta fundamental establecer puentes y alianzas entre el educador del museo y el profesor del aula, realizándose actividades previas y posteriores a la visita que complementen la visita al museo o a otros elementos patrimoniales (Méndez, 2017). Pues, no se trata simplemente de ir con el alumnado a visitar un museo, sino de vincular el estudio y el uso del patrimonio cultural con la realidad social de los escolares para desarrollar en ellos una competencia social y ciudadana crítica.

Por su parte, en el ámbito educativo no formal, el programa didáctico de los museos debe ir encaminado a despertar sensibilidades, crear actitudes favorables y ofrecer situaciones que permitan al público, sobre todo al infantil, comprender, valorar y disfrutar de las colecciones de objetos y las producciones artísticas. Como afirman Escarbajal de Haro y Martínez de Miguel (2012), desde el museo se pueden trabajar educativamente elementos tan importantes como la identidad y el sentimiento de pertenencia, todo ello desde el punto de vista del visitante, que es el auténtico protagonista, proporcionando espacios de encuentro y comunicación que favorezcan la participación ciudadana. Por ello, de modo general, se observa la necesidad de trabajos centrados en las concepciones del alumnado en relación con el patrimonio 
Estudio sobre el patrimonio de la ciudad de Lorca... - S. Martínez de Miguel y otros

lo que parece hacer aconsejable seguir investigando en este sentido para desarrollar propuestas didácticas que permitan un aprendizaje verdaderamente significativo.

\section{OBJETIVOS Y METODOLOGÍA}

\section{a. Objetivos}

La salvaguarda de los bienes culturales y naturales (conservación, gestión y difusión) se ha convertido en uno de los grandes temas de interés de nuestro tiempo. Esta circunstancia adquiere, si cabe todavía mayor relevancia en una ciudad como la de Lorca, que pese a ser una de las más importantes del patrimonio histórico artístico del Barroco Murciano, fue asolada por el mayor movimiento sísmico que ha acontecido en la Región de Murcia en los últimos años. Sin embargo, unos de los graves problemas a la hora de enfrentarse a la tutela del patrimonio lo constituye la escasa valoración y concienciación social que ha generado, fruto en gran medida, del desconocimiento de los propios elementos y bienes.

De hecho, a nivel legislativo Molina y Pinto (2015) indican que con la LOMCE actual se sigue produciéndose un desfase significativo entre el potencial didáctico del patrimonio para la enseñanza de las ciencias sociales y su presencia en el currículum oficial, no suponiendo la nueva ley una mejora en su tratamiento. En el caso específico de Murcia, teniendo en cuenta que, uno de los cambios introducidos con la LOMCE es la desaparición del área de conocimiento del medio, creándose en su lugar dos áreas diferenciadas, la de ciencias naturales y la de ciencias sociales, de acuerdo con el Real Decreto 198/2014, de 5 de septiembre, por el que se establece el currículo de la Educación Primaria en la Comunidad Autónoma de la Región de Murcia (CARM), el área en la que se trabaja el patrimonio cultural, es la de ciencias sociales. Además los autores indican, que las referencias directas al término patrimonio/patrimonial en el currículum oficial de la CARM, se encuentran de forma mayoritaria en el área de expresión artística y no en el área de Ciencias Sociales que, como se ha indicado, es la encargada de mostrar la importancia que tienen los bienes culturales como legado del pasado.

Atenordeloexpuesto,pareceexistirundesfaseentreelusoderecursospatrimoniales en los centros educativos (muy por debajo de lo deseado) y su presencia en el currículum oficial de educación primaria. Existiendo ese desfase, se ha considerado importante saber, lo que los escolares de sexto de primaria de Lorca conocen del patrimonio y los museos de su entorno, su opinión y expectativas al respecto, para identificar posibles áreas de actuación y mejora que permita ofrecerles experiencias satisfactorias.

Por ello el estudio parte de un objetivo general que es determinar el conocimiento de los bienes culturales de su ciudad que tiene el alumnado de sexto curso de educación primaria de Lorca. Para ello, el estudio plantea dos objetivos específicos: 
Revista de Humanidades, 38 (2019). p.11-36. ISSN 1130-5029

1. Identificar cuáles son los elementos patrimoniales conocidos por el alumnado, así como elcontexto en el que fueron conocidos.

2. Analizar las variables socioeducativas que están influyendo sobre el conocimiento del patrimonio por parte de los escolares.

\section{b. Participantes}

La muestra objeto de estudio está formada por un total de 156 estudiantes. Para su selección, se utilizó una técnica de muestreo no probabilística. Se llevó a cabo un muestreo deliberado o por conveniencia, utilizando como único criterio el que fueran alumnos de $6^{\circ}$ de Primaria de los 31 colegios públicos de la ciudad de Lorca (Murcia), puesto que así los datos recogidos indicarían las características de este conocimiento al terminar la etapa educativa. Debido a las limitaciones en la selección de la muestra, el estudio que se expone, tiene un carácter exploratorio y no extrapolable. No obstante, se considera que presenta el perfil característico del universo sociocultural que se quería estudiar. Entre las características sociodemográficas de la muestra (ver Tabla 1), cabe destacar que la edad media de los participantes fue de 12.61 años $(\mathrm{DT}=0,49)$, siendo el estudiante más joven de 11 años y el mayor de 13 años, $\mathrm{y}$ existiendo práctica paridad en cuanto al género.

Tabla 1. Características de la muestra seleccionada de estudiantes $(\mathrm{n}=156)$

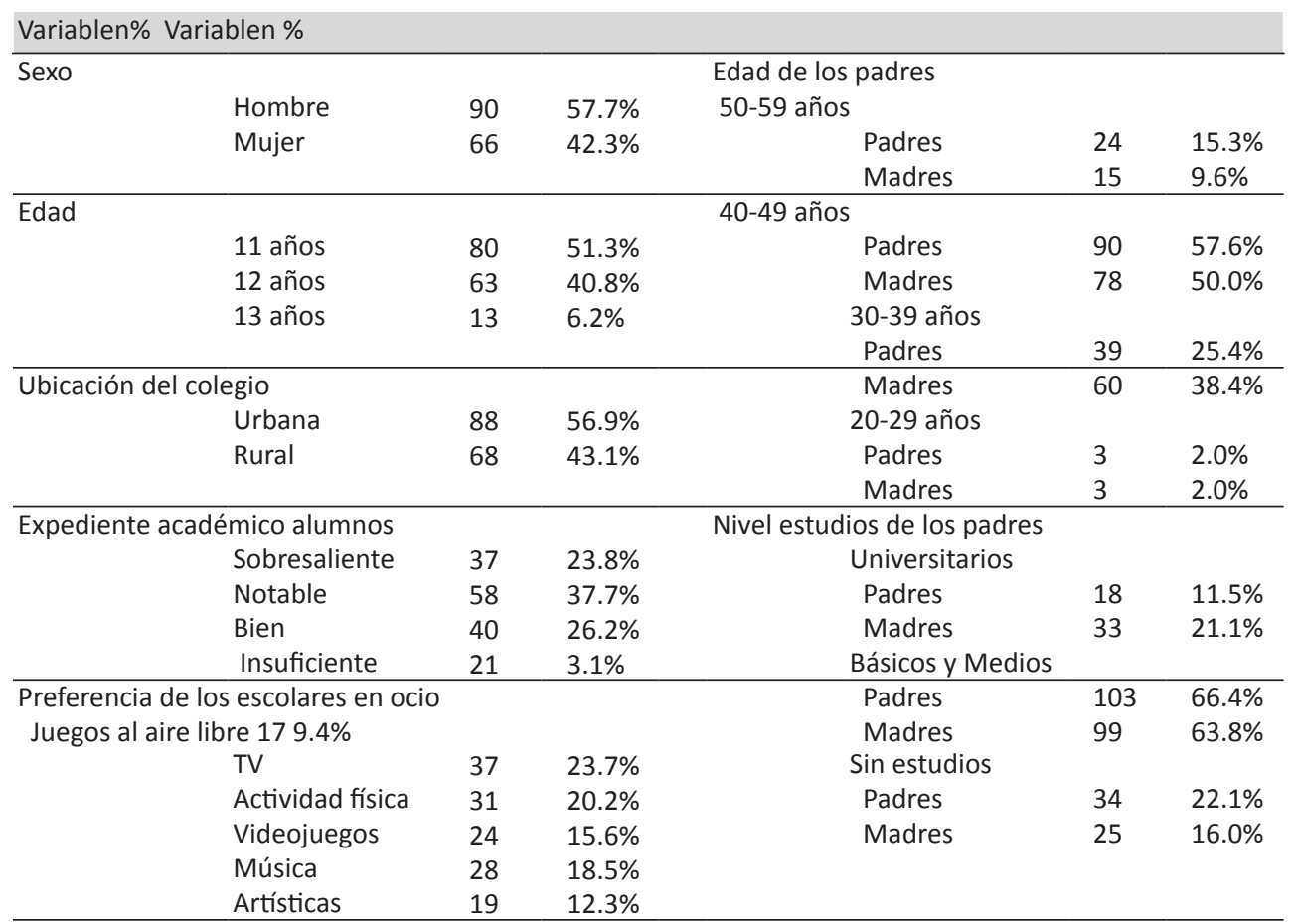

Fuente: elaboración propia 
Estudio sobre el patrimonio de la ciudad de Lorca... - S. Martínez de Miguel y otros

c. Instrumento

Para la recolección de datos, se ha utilizado como instrumento de recogida de información un cuestionario creado ad hoc. El cuestionario dirigido a los escolares de $6^{\circ}$ curso, se estructura en cuatro bloques: $1^{\circ}$ ) presentación e instrucciones; $2^{\circ}$ ) datos de identificación y sociodemográficos; $3^{\circ}$ ) cuerpo de preguntas organizadas en cinco bloques de contenidos: calificaciones académicas, ocio y tiempo libre, datos de sus progenitores, importancia del patrimonio histórico artístico; relación del patrimonio arquitectónico de Lorca (conocimiento, visita, contexto de la visita y expectativas de volver a visitarlo); $4^{\circ}$ ) agradecimientos.

Para armar la validez del cuestionario, se llevó a cabo el siguiente proceso:

1. Diseño inicial del cuestionario, y posterior consideración cualitativa por parte de los investigadores $(n=3)$.

2. Revisión de las preguntas, a partir del juicio de expertos externos a la investigación, a través de una valoración interjueces, contando con la participación de 3 profesionales pertenecientes a los ámbitos disciplinares de Métodos de Investigación y Diagnóstico en Educación y Teoría e Historia de la Educación de la Universidad de Murcia, así como dos técnicos municipales de patrimonio de la ciudad.

3. Tras la cumplimentación de la escala de valoración cualitativa realizada por los jueces externos $(\mathrm{n}=5)$, y a través de las observaciones que se consideraron necesarias para mejorar, modificar, eliminar o incorporar nuevas propuestas, se redefinieron 5 de los ítems para mejorar su claridad y facilitar la comprensión de los mismos, se añadieron 4 ítems nuevos, no contemplados en el cuestionario original, que hacían referencia a los datos generales y se eliminaron 6 ítems relacionados con los elementos patrimoniales y museos entendiendo, que el cuestionario era así, más adecuado.

4. Elaboración definitiva del cuestionario, que se adjunta como anexo consultable de esta investigación.

\section{d. Procedimiento de recogida y análisis de datos}

Una vez sometido a la validación a través del juicio de los expertos, se solicitó el consentimiento informado de los padres, y se procedió a su aplicación a los alumnos de forma colectiva. Para ello, en primer lugar se contactó con los centros educativos para poder informar sobre la realización de este estudio. Se informó al alumnado sobre la participación en el estudio, se les comunicó oralmente y por escrito, que la participación era voluntaria y, además, se les informó que podían rehusar a participar en el estudio en cualquier momento. El cuestionario fue entregado para que lo cumplimentaran y tuvo una duración media de 40 minutos. 
Revista de Humanidades, 38 (2019). p.11-36. ISSN 1130-5029

El análisis de los datos se ha realizado con el programa estadístico SPSS, versión 22 para Windows, en el que las diferencias consideradas estadísticamente significativas son aquellas cuya $\mathrm{p}<0.05$.Para la obtención de los resultados se han realizado análisis general de las variables, empleando métodos descriptivos básicos. De la misma manera, se han empleado estudios de asociación entre variables, con la finalidad de conocer si las variables estudiadas mostraban algún tipo de relación entre ellas, y el conocimiento del patrimonio cultural y museístico, para lo que se ha utilizado el estadístico chi cuadrado de Pearson para variables categóricas.

\section{RESULTADOS}

En cuanto al conocimiento del patrimonio local y los museos de la ciudad de Lorca, objetivo principal de este trabajo de investigación, de manera prácticamente unánime y mayoritaria (97.7\%), los escolares otorgan, con respuestas afirmativas, la importancia de conocer los museos y monumentos de su ciudad, como herramienta de adquisición de cultura y conocimiento de la historia a través de su visita. En este sentido, y en relación con los distintos elementos patrimoniales seleccionados, de forma mayoritaria los escolares desean visitar, o volver a visitar en el caso de que lo hayan hecho, todos los elementos patrimoniales, siendo el elemento con mayores expectativas de visita, el Teatro Guerra (92.3\%), seguido de la Fortaleza del Sol $(89.2 \%)$.

En relación con el uso o utilidad del patrimonio, se puede observar la visita de determinados elementos arquitectónicos debido a la realización de actividades puntuales en su interior, tales como exposiciones, representaciones artísticas, etc. En relación con las visitas a los elementos patrimoniales (Gráfico 1), el Teatro

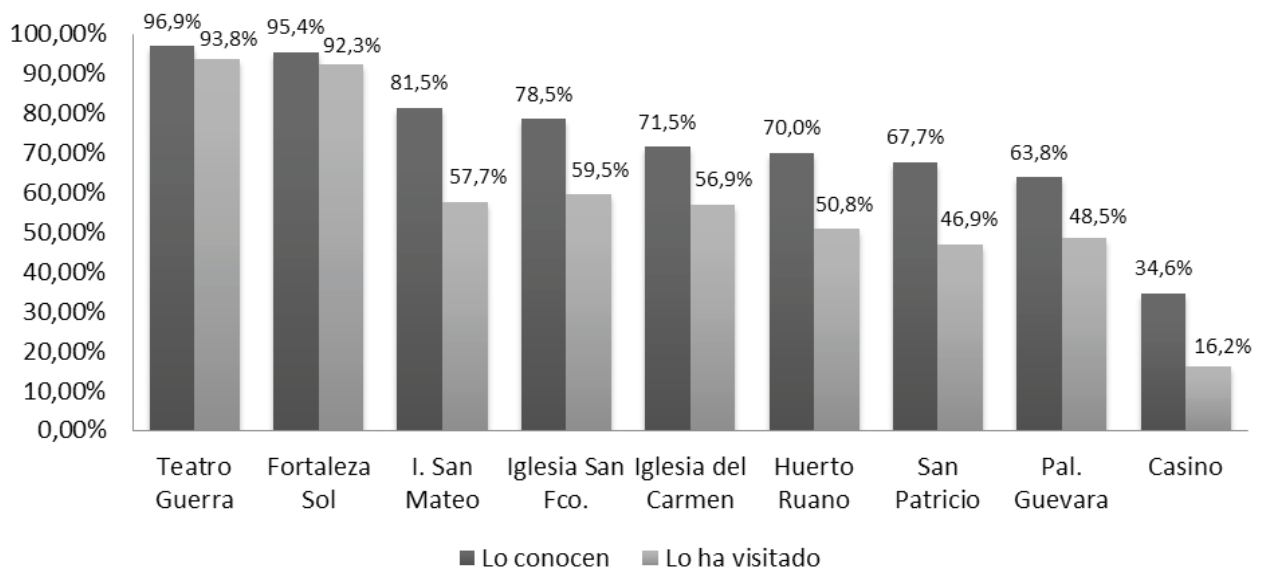

Gráfico 1. Conocimiento y visita del patrimonio cultural Lorquino por parte del alumnado. Fuente: elaboración propia. 
Estudio sobre el patrimonio de la ciudad de Lorca... - S. Martínez de Miguel y otros

"Guerra" es el lugar más visitado por los alumnos debido a su uso como espacio de representaciones culturales, siendo muy limitadas las referencias que hacen los estudiantes a su valor como monumento histórico. La Fortaleza del Sol es el segundo elemento más visitado. Este castillo, símbolo de la ciudad, se ha convertido en un importante recurso turístico a través del proyecto "Lorca Taller del Tiempo". Las visitas al resto de elementos del patrimonio cultural, se vinculan de forma mayoritaria al uso al que el bien patrimonial está destinado, independientemente de su valor artístico, histórico y/o cultural.

De acuerdo con los datos analizados, como se puede observar en la Tabla 2, en líneas generales, los escolares visitan mayoritariamente los elementos patrimoniales acompañados de sus padres, con un promedio elevado (66.3\%). En segundo lugar lo hacen en compañía de la institución educativa del colegio en proporciones considerablemente inferiores $(14.0 \%)$. Sin embargo, hay que destacar que los elementos del patrimonio más visitados son el Teatro Guerra (93.8\%), y la Fortaleza del Sol (92.3\%), fueron también visitados en compañía de la institución educativa del colegio con porcentajes más elevados $(28.6 \%, 22.5 \%$, respectivamente). De manera minoritaria, la visita al patrimonio cultural de la ciudad tiene lugar cuando son acompañados de otros familiares, o de amigos, etc., (12.2\%).

Datos similares fueron encontrados en lo que se refiere a las entidades museísticas, donde los escolares acuden, como elemento más representativo, al Centro de Visitantes (46.9\%), siendo esta experiencia de tipo familiar. En segundo lugar lo hacen en compañía del colegio, con una excepción, la del Museo Arqueológico, que ha sido más visitado en compañía del colegio (45.3\%), que en compañía de los padres $(43.7 \%)$.

No obstante, se observa que los museos que tienen una identidad definida en torno a los Museos de Bordados, y que pertenecen a las distintas cofradías de Semana Santa, son de manera amplia y mayoritaria, los más visitados por parte de los alumnos. Así, el más visitado fue el Museo del Paso Azul (76.9\%), seguido del Museo del Paso Blanco (76.2\%), y del Museo Arqueológico (66.9\%).

En cuanto a la forma de realizar la visita, cabe señalar que, existen dos de ellos que se visitan en exclusiva en compañía de sus padres, como son el Museo del Paso Encarnado (82.9\%), y el Museo del Paso Morado (76.1\%).

Los datos encontrados en lo que se refiere a las entidades museísticas (Gráfico 2), son similares a las obtenidas respecto al patrimonio. No obstante, cabe destacar que el porcentaje de la muestra de alumnado que desconoce los museos $(37.9 \%)$, es superior, al porcentaje de alumnos que desconoce el patrimonio $(26.7 \%)$. De la misma manera, se mantienen las diferencias en lo que se refiere a las visitas. Así cabe destacar que el porcentaje de estudiantes que no han visitado el patrimonio lorquino $(41.9 \%)$ es inferior, al de aquellos que nunca han visitado alguno de los museos $(55.1 \%)$. 
Revista de Humanidades, 38 (2019). p.11-36. ISSN 1130-5029

Tabla 2. Visita de los escolares al patrimonio cultural y museístico de Lorca (N=156)

\begin{tabular}{|c|c|c|c|c|c|}
\hline Institución & $\mathrm{n}$ & $\%$ & Institución & $\mathrm{n}$ & $\%$ \\
\hline Teatro Guerra & & & Museo Paso Blanco & & \\
\hline Padres & 54 & $36.8 \%$ & Padres & 63 & $63.8 \%$ \\
\hline Colegio & 42 & $28.6 \%$ & Colegio & 15 & $15.6 \%$ \\
\hline Ambos & 38 & $25.4 \%$ & Ambos & 4 & $4.8 \%$ \\
\hline Otros & 13 & $9.0 \%$ & Otros & 15 & $15.6 \%$ \\
\hline Fortaleza del Sol & & & Museo Paso Azul & & \\
\hline Padres & 49 & $34.1 \%$ & Padres & 66 & $77.7 \%$ \\
\hline Colegio & 32 & $22.5 \%$ & Colegio & 7 & $8.3 \%$ \\
\hline Ambos & 56 & $38.3 \%$ & Ambos & 2 & $2.7 \%$ \\
\hline Otros & 7 & $5.0 \%$ & Otros & 9 & $11.1 \%$ \\
\hline Iglesia de San Mateo & & & Museo Arqueológico & & \\
\hline Padres & 94 & $85.3 \%$ & Padres & 33 & $43.7 \%$ \\
\hline Colegio & 0 & $0 \%$ & Colegio & 34 & $45.3 \%$ \\
\hline Ambos & 0 & $0 \%$ & Ambos & 5 & $7.8 \%$ \\
\hline Otros & 16 & $14.6 \%$ & Otros & 2 & $3.1 \%$ \\
\hline Iglesia de San Francisco & & & Centro de Visitantes & & \\
\hline Padres & 84 & $81.8 \%$ & Padres & 27 & $46.9 \%$ \\
\hline Colegio & 0 & $0 \%$ & Colegio & 22 & $38.7 \%$ \\
\hline Ambos & 2 & $2.5 \%$ & Ambos & 7 & $12.2 \%$ \\
\hline Otros & 16 & $15.5 \%$ & Otros & 1 & $2.0 \%$ \\
\hline Iglesia del Carmen & & & Museo Paso Morado & & \\
\hline Padres & 93 & $93.0 \%$ & Padres & 38 & $76.1 \%$ \\
\hline Colegio & 0 & $0 \%$ & Colegio & 0 & $0 \%$ \\
\hline Ambos & 0 & $0 \%$ & Ambos & 0 & $0 \%$ \\
\hline Otros & 7 & $7,0 \%$ & Otros & 11 & $23.8 \%$ \\
\hline Huerto Ruano & & & Museo Paso Encarnado & & \\
\hline Padres & 34 & $37.8 \%$ & Padres & 40 & $82.9 \%$ \\
\hline Colegio & 33 & $36.3 \%$ & Colegio & 0 & $0 \%$ \\
\hline Ambos & 12 & $13.6 \%$ & Ambos & 0 & $0 \%$ \\
\hline Otros & 11 & $12.1 \%$ & Otros & 9 & $17 \%$ \\
\hline \multicolumn{6}{|l|}{ San Patricio } \\
\hline Padres & 61 & $83.6 \%$ & & & \\
\hline Colegio & 0 & $0 \%$ & & & \\
\hline Ambos & 0 & $0 \%$ & & & \\
\hline Otros & 11 & $16.3 \%$ & & & \\
\hline \multicolumn{6}{|l|}{ Palacio de Guevara } \\
\hline Padres & 56 & $74.6 \%$ & & & \\
\hline Colegio & 3 & $4.7 \%$ & & & \\
\hline Ambos & 3 & $4.7 \%$ & & & \\
\hline Otros & 11 & $15.8 \%$ & & & \\
\hline \multicolumn{6}{|l|}{ Casino } \\
\hline Padres & 19 & $76.1 \%$ & & & \\
\hline Colegio & 2 & $9.5 \%$ & & & \\
\hline Ambos & 0 & $0 \%$ & & & \\
\hline Otros & 3 & $14.2 \%$ & & & \\
\hline
\end{tabular}

Fuente: elaboración propia. 


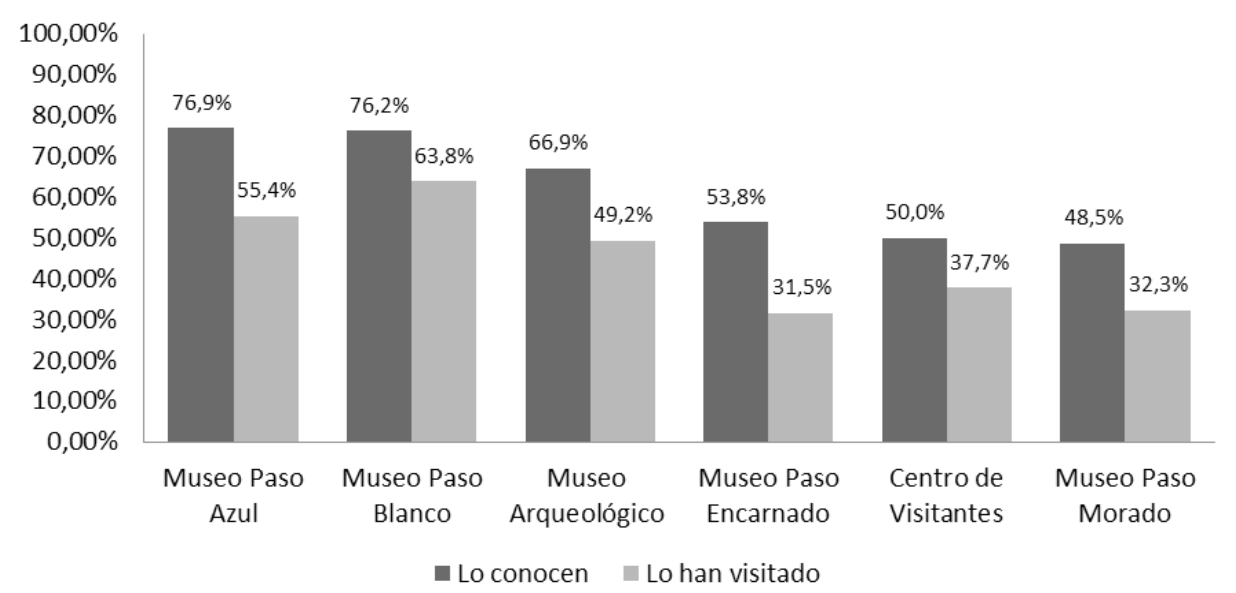

Grafico 2. Conocimiento y visitas de los escolares a los museos.

Fuente: elaboración propia

En su conjunto, el promedio de escolares que desconocen el patrimonio de Lorca seleccionado, se considera elevado (32.2\%).

En cuanto a los elementos patrimoniales, los más conocidos por los escolares son el Teatro Guerra, la Fortaleza del Sol, la Iglesia de San Mateo y la Iglesia de San Francisco. Por el contrario, los elementos menos conocidos son el Casino Artístico Literario y el Palacio de Guevara. El porcentaje de aquellos estudiantes que no lo han visitado nunca, alcanza prácticamente a la mitad de la muestra seleccionada $(48.2 \%)$.

En cuanto a la asociación entre las diversas variables analizadas y el conocimiento del patrimonio Lorquino por parte del alumnado, se encontraron diferencias en la variable género (Palacio de Guevara, $\chi^{2}=4.541, p=.033$, Iglesia de San Patricio, $\chi^{2}=$ $5.626, p=.018$, y Huerto Ruano, $\chi^{2}=8.064, p=.005$ ). De acuerdo con los resultados obtenidos, se señala la relación existente que se ha encontrado entre las variables sexo y el deseo, o no, de volver a visitar el patrimonio histórico y cultural señalado. Así, se encontró asociación estadísticamente significativa para las variables sexo masculino y la falta de deseo de volver a visitar el elemento patrimonial.

Considerando la influencia del nivel cultural de los padres en el conocimiento del patrimonio y los museos locales de sus hijos, se encontraron diferencias entre las diversas entidades del patrimonio $\left(\chi^{2}=11.332, \mathrm{p}=.045\right)$. Realizando un desglose de los resultados obtenidos, en función del nivel de estudios alcanzado por los progenitores, concretamente por la figura paterna, se observan diferencias estadísticamente significativas para algunos elementos del patrimonio cultural de la ciudad de Lorca. Así, la representación del nivel de estudio del padre y por ejemplo el conocimiento de la Iglesia del Carmen evidencia gráficamente esta situación (Gráfico 3). 


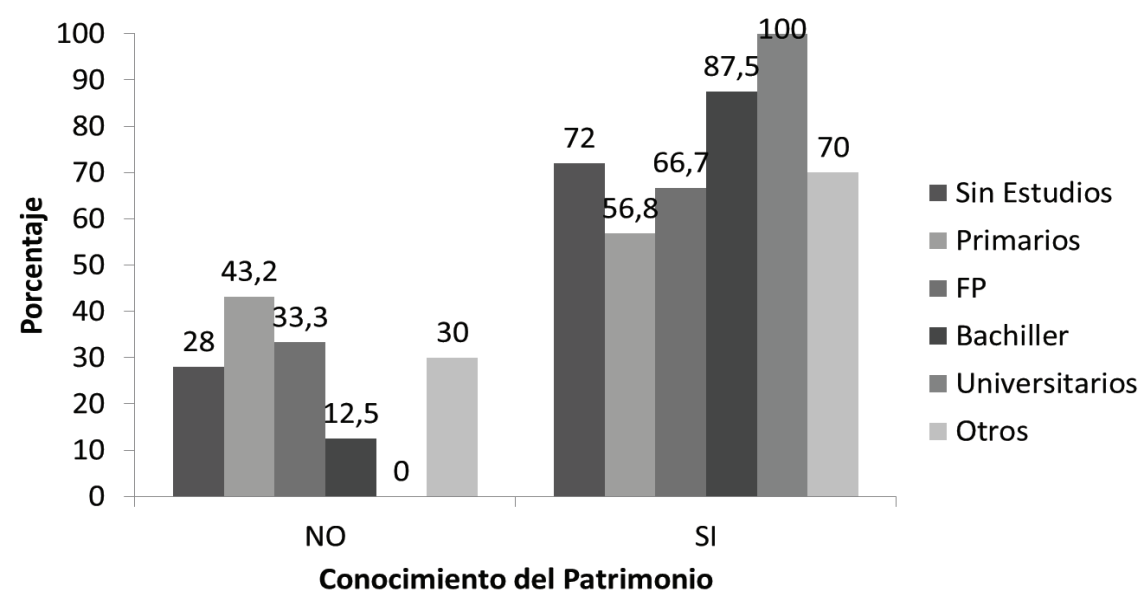

Gráfico 3. Diagrama de barras de la frecuencia del conocimiento del patrimonio en cuanto a la variable nivel de estudios del padre.

Fuente: elaboración propia

En cuanto a los resultados obtenidos en el caso del mayor nivel de formación adquirido por parte de las madres (Gráfico 4), destacan los resultados estadísticamente significativos, que se asociaron con el deseo de volver a visitar el museo Nicolás Salzillo (Paso Morado) $\chi^{2}=14.798, p=.011$.

Haciendo una extrapolación cautelosa a la población representada, cabe destacar, que los datos señalan la existencia de relación significativa entre las variables conocimiento del patrimonio y nivel sociocultural de los progenitores, así como con la frecuencia de visitas. De tal manera que en su conjunto, el mayor grado de cualificación de los padres (ya sea padre o madre) fue asociado a un mayor

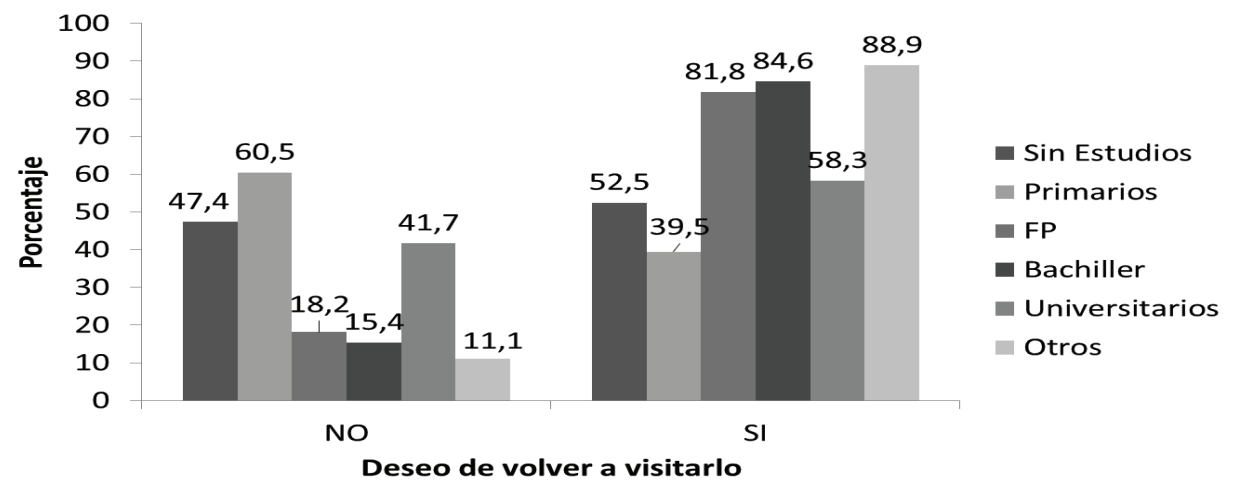

Gráfico 4. Diagrama de barras de la frecuencia de representación del deseo de volver a visitar el patrimonio en cuanto a la variable nivel de estudios de la madre. Fuente: elaboración propia. 
Estudio sobre el patrimonio de la ciudad de Lorca... - S. Martínez de Miguel y otros

conocimiento del patrimonio cultural, además de la mayor frecuencia de visitas, y deseo de volver a visitarlos.

Respecto a la variable ubicación geográfica del centro educativo, clasificados en zona urbana y zona rural, en su conjunto los resultados obtenidos (Museo Salzillo, $\chi^{2}(1)=6.400, p=.011$; Huerto Ruano, $\chi^{2}(1)=12.644, p=.000$; Iglesia del Carmen, $\left.\chi^{2}(1)=3.947, p=.047\right)$, se observa una relación significativa entre la variable ubicación geográfica, ámbito urbano, y el mayor conocimiento de museos y del patrimonio cultural. De tal manera que la ubicación del colegio en el ámbito urbano se asoció también a una mayor frecuencia de visita de los museos y monumentos, que los que se ubicaron en zonas rurales.

\section{DISGUSIÓN}

Los resultados muestran que hay todavía mucho por hacer en el ámbito de la educación patrimonial para que el patrimonio histórico artístico Lorquino sea un recurso al servicio de la educación de los estudiantes de primaria, y se aproveche realmente todo su potencial, pues se evidencia un escaso conocimiento de sus bienes culturales. Esto, a pesar de considerar el 97,7\% de los escolares participantes en el estudio, que es importante conocerlo, y mostrar de forma mayoritaria, una predisposición favorable a su visita, asociándola a aspectos culturales, asi como a un mayor conocimiento de su ciudad. Esta deficiencia también se ha podido constatar en otros trabajos de investigación realizados en torno a la educación patrimonial (Castellón y Martínez, 2001; Izquierdo López y Prados, 2014). En cualquier caso, la mayoría de estos estudios abordan el proceso de investigación desde una perspectiva distinta, centrándose fundamentalmente en el análisis de la legislación educativa y patrimonial, el análisis de los libros de texto y los materiales didácticos empleados en centros educativos o museos, y en el estudio de las concepciones de los gestores patrimoniales y el profesorado que pueden tener entre sus competencias educativas la enseñanza del patrimonio.

De acuerdo con los resultados obtenidos en la investigación, los participantes en el estudio presentan un conocimiento, que se puede calificar como medio-bajo del patrimonio local y museístico de Lorca. Además, este conocimiento suele ser vago e impreciso, utilizándose para su descripción adjetivos muy genéricos como "grande, chulo, bonito, interesante o aburrido", siendo el porcentaje de alumnado que los han visitado reducido, y referidos a elementos patrimoniales muy concretos. Estudios previos (Reyes, 2016; Ministerio de Cultura, 2011), han mostrado una menor presencia de la población infantil, que de adultos. También, que en el caso de las visitas de público infantil, éstas se realizan con mayor frecuencia dentro del entorno familiar que del escolar, lo que concuerda con lo observado en el estudio.

Los datos hallados en la presente investigación están en consonancia con otras, que en las últimas décadas se están llevando a cabo desde las áreas de Didáctica de 
Revista de Humanidades, 38 (2019). p.11-36. ISSN 1130-5029

las Ciencias Sociales de distintas universidades españolas, que abordan su estudio fundamentalmente a partir de las concepciones de los docentes, el alumnado, los materiales didácticos y el análisis de la legislación educativa y patrimonial. Así, investigaciones recientes (Calaf, San Fabián y Gutiérrez, 2017; Fontal, et al., 2017; López Cruz, 2014), ponen de manifiesto la existencia de un desfase significativo entre el potencial didáctico del patrimonio para la enseñanza de las Ciencias Sociales, y su presencia en las aulas, al no vincularse con una valoración del patrimonio cultural como herencia del pasado, que genere actitudes de compromiso alrededor de su protección y puesta en valor de la identidad de un pueblo.

Uno de los resultados obtenidos en este sentido, la menor frecuentación del patrimonio cultural a través del entorno escolar, podría atribuirse entre otras cuestiones, a las dificultades de involucrar al profesorado, al alumnado, al propio centro de enseñanzas, además de los departamentos de Educación y Acción cultural de los museos, en una actividad didáctica integral que pueda conseguir desarrollar competencias curriculares, que no se podrían adquirir exclusivamente en el aula. Sin embargo, la investigación ofrece resultados diferentes en un caso singular como es la visita al Museo Arqueológico de la ciudad, que entronca en la línea de otros estudios similares, que muestran la mayor asistencia por parte de los estudiantes de primaria a museos de tipo científico (Izquierdo, López y Prados, 2014).

A pesar del mayor grado de conocimiento de los bienes culturales favorecidos desde el entorno familiar, a tenor de los resultados obtenidos, parece también necesario mejorar la implicación de las familias con proyectos que despierten su interés y permitan superar las desigualdades observadas, entendiendo que no todas tienen las mismas oportunidades de conocer los bienes culturales de su ciudad. En este sentido y referido al nivel sociocultural de los padres, respecto del conocimiento del patrimonio y los museos locales, los análisis de asociación entre variables ponen de manifiesto la existencia de un número considerable de relaciones entre el conocimiento del patrimonio y los museos locales, con el nivel cultural de los padres. Haciendo un juicio prudencial a la población que pudiera estar representada, pareciera existir una posible relación que indica que un mayor grado de cualificación de los progenitores (ya sea padre o madre), pudiera estar relacionado con un mayor conocimiento del patrimonio cultural. También la cualificación de los progenitores parece relacionarse de manera positiva con la frecuencia de visitas y el grado de conocimiento por parte de los hijos. Esta casuística, va en la línea de los resultados obtenidos en la revisión de estudios que realizaron Guisasola y Morentin (2007). En base a ello, estos mismos autores, proponen la necesidad de que se aumenten las visitas desde el ámbito escolar, para suplir las lagunas que puedan tener los niños en su entorno familiar, evitando así las desigualdades. En este sentido, investigaciones más recientes (Estepa, 2013; Molina y Pinto, 2015), concluyen que los profesores podrían realizar una gran variedad de prácticas a través del contacto directo con el patrimonio local superando así los déficits existentes por parte de la administración educativa, editoriales, docentes y gestores patrimoniales. 
Estudio sobre el patrimonio de la ciudad de Lorca... - S. Martínez de Miguel y otros

Particularizando en los datos encontrados en función del género del alumnado, diversos estudios en población general, han mostrado un mayor interés y frecuencia de visita a museos, en el caso de las niñas, que en el caso de los niños (Ministerio de Educación, 2015). En la línea de dichas investigaciones, los resultados del presente estudio muestran que existe un menor deseo de volver a visitar el patrimonio histórico y cultural, asociado a la variable sexo masculino. Es difícil vislumbrar una razón o justificación a este hecho. Sin embargo, parece apuntar a las distintas preferencias que muestran niños y niñas a la hora de elegir actividades de ocio y tiempo libre, así como también, por ejemplo a su grado de motivación hacia la realización de otras actividades, como puede ser la lectura en términos generales, o concretamente, su actitud hacia la lectura social o colectiva (Artola, Sastre y Barraca, 2017).

En cuanto al sentimiento identitario de los estudiantes de educación primaria de la ciudad de Lorca con determinados bienes patrimoniales vinculados, en función de los resultados obtenidos, se observa una estrecha relación, sobre todo, con los relacionados en la celebración de la Semana Santa y que se caracteriza por sus Desfiles Bíblico-Pasionales y sus bordados. No obstante ello, la rivalidad existente en la ciudad de Lorca entre los "Pasos", sobre todo entre los dos mayoritarios, Blanco y Azul, provoca que ese fuerte sentimiento de apego hacia determinados bienes suponga, al mismo tiempo, el escaso interés de los escolares por los bienes relacionados con el resto de cofradías, cuestión sobre la que habría que reflexionar, siendo considerados una importante tradición y manifestación cultural transmitida de generación en generación. Esto supone, que los profesionales de la educación disponen de un gran número de espacios patrimoniales de carácter religioso de cuyo valor potencial como recurso didáctico no deberían prescindir (Gónzalez Monfort y Magoga, 2014; Martínez Gil, 2014). No obstante, las investigaciones indican que también es necesaria la formación del profesorado en contenidos patrimoniales, como identidad y apropiación ciudadana de los bienes culturales, para que de esta manera se facilite la transmisión de conocimientos y pueda existir mayor sensibilización (Fontal et al., 2017; Méndez, 2017). De la misma manera quizás en este punto es necesario hacer mención a la necesidad de coordinar los distintos ámbitos educativos tal y como se recoge en el Plan Nacional de Educación y Patrimonio (2013), y replantear la metodología de la formación de los estudiantes de Magisterio en lo que a enseñanza patrimonial se refiere, tal y como apunta Ávila y Duarte (2017). En este sentido, la animación sociocultural como metodología y herramienta educativa que traspasa los límites de la educación formal, presenta múltiples posibilidades y hace posible el diálogo intercultural en relación con el patrimonio y la identidad. Además, el marco de la Animación Sociocultural es ideal para desarrollar propuestas inclusivas en torno al patrimonio cultural, al devolver la voz a la comunidad y ser ésta la que diseña sus propios programas de intervención sociocomunitaria (Escarbajal y Martínez de Miguel, 2012). De esta forma, las ciudades pasan a ser concebidas como grandes espacios educativos donde se promueven metodologías de trabajo participativas y se otorga una especial atención a aquellos colectivos que se 
Revista de Humanidades, 38 (2019). p.11-36. ISSN 1130-5029

encuentren en riesgo de exclusión social. De la misma manera, también ayudaría en este sentido, el cambio de enfoque en los decretos legislativos y curriculum vigentes en educación, hacia una acción más profunda, plural, relevante, y menos adoctrinante, tal y como afirman Escribano y Molina (2015a, p.22): "cuando se habla de museos, parece ser más importante concienciar al alumno acerca de cómo ha de comportarse en ellos y hacerles ver que allí se puede apreciar y disfrutar la cultura, que mostrar que en estos se puede ampliar lo aprendido en clase, y sobre todo combinarlo con esas clases". Cómo se deriva del análisis realizado diversos serían los factores a tener en consideración para mejorar el nivel de conocimientos de los escolares acerca del patrimonio de su ciudad: curriculum y decretos educativos, familias, instituciones museísticas, formación de los profesionales, y metodologías educativas.

\section{GONCLUSIONES}

Este trabajo pretende determinar el conocimiento que los escolares de sexto de primaria tenían sobre determinados elementos de los bienes culturales de la ciudad de Lorca, con el objetivo de contar con un punto de partida a partir del cual poder desarrollar propuestas didácticas en torno a la educación patrimonial. Con carácter general y en relación con la mayoría de elementos patrimoniales y museos, los alumnos valoran positivamente la visita a los mismos. No obstante, en relación a este objetivo general se puede decir que el trabajo de investigación ha puesto de relieve que los escolares participantes en el estudio tienen un conocimiento limitado del patrimonio cultural local, centrándose las visitas en determinados elementos y museos. El deseo de visitar los elementos patrimoniales y los museos locales en los escolares vendría determinado por la existencia de una visita anterior positiva o por las expectativas de aprender y divertirse generadas por los comentarios de amigos, compañeros o familiares. Los resultados también han puesto en evidencia la necesidad de utilizar estrategias didácticas creativas e innovadores que despierten el interés de los niños y de adaptar las visitas guiadas al público infantil.

En respuesta al primer objetivo específico planteado, identificar cuáles son los elementos patrimoniales conocidos por el alumnado, así como el contexto en el que fueron conocidos, cabe destacar que en relación con los edificios y monumentos de esta investigación, el mayor o menor conocimiento de los mismos viene determinado por el uso al que se dedica el bien y, solo en determinados casos, se identifica con el valor identitario del elemento en cuestión. Así, en relación con las visitas a los elementos patrimoniales, el Teatro Guerra es el lugar más visitado por los alumnos debido a su uso como espacio de representaciones culturales, siendo muy limitadas las referencias que hacen los estudiantes a su valor como monumento histórico. La Fortaleza del Sol es el segundo elemento más visitado. Este castillo, símbolo de la ciudad, se ha convertido en un importante recurso turístico. Respecto de los lugares de culto asociados con el patrimonio religioso, se observa que los escolares 
Estudio sobre el patrimonio de la ciudad de Lorca... - S. Martínez de Miguel y otros

conocen y visitan este tipo de edificios al acudir a ellos con motivo de la celebración de determinados eventos, no apreciándose la existencia de otros valores culturales asociados. En este sentido, dada la amplitud del patrimonio religioso de la ciudad de Lorca, sería conveniente desarrollar fórmulas para su difusión desde una perspectiva histórica e identitaria. Respecto a los museos, los más conocidos y visitados son el Museo de Bordados del Paso Azul y el Museo de Bordados del Paso Blanco. El caso de los museos de bordados pertenecientes a las distintas cofradías de Semana Santa es similar al de los lugares de culto asociados a las mismas: su conocimiento y visita se vincula con la pertenencia o sentimiento de afecto hacia una determinada cofradía. En este contexto, se presume un matiz excluyente que se manifiesta fundamentalmente en relación con las dos cofradías principales y que deriva del fuerte sentimiento de apego hacia determinados bienes. Teniendo en cuenta lo anterior, se considera conveniente que desde las administraciones y las instituciones (escolares y patrimoniales), se potencie la visita a estos museos independientemente de la afiliación a una determinada cofradía.

Respecto al contexto en el que son introducidos los escolares en los bienes culturales de Lorca, señalar que se producen principalmente a través de visitas familiares, quedando las visitas escolares reducidas a determinados elementos muy concretos. Así, en relación con las visitas que los estudiantes realizan mayoritariamente con sus progenitores son a las Iglesias, con motivo de la asistencia a eventos de tipo religioso.

Respecto al segundo objetivo específico planteado en esta investigación, analizar las variables socioeducativas que están influyendo sobre el conocimiento del patrimonio por parte de los escolares, resulta interesante destacar la existencia de algunas variables moduladoras que podrían estar influyendo en las diferencias encontradas respecto a la mayor o menor familiaridad de los escolares con los bienes patrimoniales de su ciudad. Así, del conjunto de variables estudiadas (edad, género, ubicación del colegio, expediente académico, preferencias de ocio, edad de los padres y nivel de estudios), existe un número considerable de relaciones entre el conocimiento del patrimonio y los museos locales, asociados al nivel cultural de los padres. Esto parece aconsejar el aumento de las visitas escolares para suplir las lagunas que puedan tener los niños en su entorno familiar evitando así las desigualdades. En cualquier caso, parece aconsejable que los proyectos que se promuevan desde las administraciones o las instituciones en relación con el patrimonio vayan acompañados de campañas de sensibilización y motivación a las familias, instituciones escolares, etc. prestando especial atención a las pedanías más rurales del municipio.

En conclusión, se puede decir que, habiendo comprobado que la educación (tanto formal como no formal) no está aprovechando suficientemente el potencial educativo del patrimonio Lorquino sería necesaria una mayor integración y un trabajo en red entre las distintas administraciones públicas para favorecer una mayor 
Revista de Humanidades, 38 (2019). p.11-36. ISSN 1130-5029

proyección comunitaria del patrimonio local. Esto no implica que no sea un recurso presente en las aulas. Existen profesionales en la escuela que utilizan el patrimonio cultural como un recurso a partir del cual potenciar la construcción de una identidad ciudadana responsable y el desarrollo de un pensamiento social crítico planteando alternativas a los problemas sociales relevantes para mejorar su futuro, pero aún no es una práctica generalizada y por ello es necesario demandarla.

Finalmente, cabe reseñar de manera muy sucinta y a modo de línea de futuro, las que podrían considerarse como propuestas o recomendaciones a nivel sociodidáctico parar favorecer y dinamizar el desarrollo sostenible del patrimonio Lorquino. Para ello se propone el desarrollo de estrategias o programas de educación patrimonial durante esta etapa educativa reconociendo que no todos los escolares tienen las mismas posibilidades de conocer y visitar el patrimonio. Dichas propuestas pueden articularse a partir de la proyección comunitaria del patrimonio. Esto es, con proyectos de intervención que no solo afecten a la escuela, sino que impliquen a todos los agentes sociales: administración local, museos, centros educativos, padres, etc. enmarcando estas acciones en el contexto de la Animación Sociocultural y la educación intercultural, y prestando especial atención a los colectivos en riesgo de exclusión social. Como punto de partida al desarrollo de este tipo de proyectos se propone la adhesión a la Asociación Internacional de Ciudades Educadoras, entrando a formar parte activa de este conjunto de ciudades cuyo objetivo común es trabajar conjuntamente en proyectos y actividades para mejorar la calidad de vida de sus habitantes. Desde esta perspectiva se considera importante aumentar las visitas de estos niños al patrimonio local y los museos puesto que, el conocimiento de estos elementos, además de constituir un puente entre pasado y presente les permiten conocer la ciudad en la que viven y dominar su entorno. También es necesario implicar a las familias con proyectos que despierten su interés y permitan superar las desigualdades, entendiendo que no todos tienen las mismas oportunidades de conocer su ciudad. Otro aspecto a tener en cuenta, sería la necesidad de incluir en las visitas a los monumentos que forman parte del patrimonio arquitectónico urbano, fundamentalmente en aquellas que se realicen con motivo de una exposición o actividad puntual en su interior, información específica relacionada con su importancia como elemento integrante del patrimonio cultural de la ciudad. Con ello, se podría favorecer la educación en valores (tolerancia, respeto) y a la vez se reforzaría el carácter identitario de este patrimonio. Sin embargo, desde la Animación Sociocultural no se trataría tanto de suplir carencias como de ofrecer nuevas oportunidades. En este sentido, se apostaría por el desarrollo de proyectos de intervención socioeducativos, conectando la escuela con el contexto y dando la voz a las familias para que estas aporten sus puntos de vista y propongan soluciones. En relación con los museos, es importante que éstos desarrollen programas que incluyan la visita familiar que ha de ser interactiva, participativa y con un cierto carácter lúdico, teniendo en cuenta las repercusiones que ésta puede tener en los posteriores hábitos culturales de los niños. En este sentido, es importante señalar la importancia 
Estudio sobre el patrimonio de la ciudad de Lorca... - S. Martínez de Miguel y otros

de los estudios de público en la actualidad para favorecer la inclusión social de los ciudadanos.

\section{REFERENCIAS}

Artola González, Teresa, Sastre Llorente, Santiago y Barraca Mairal, Jorge (2017). Diferencias de género en actitudes e intereses lectores. Una investigación con alumnos españoles de primaria. Bordón, 69 (1), 11-26. https://doi.org/10.13042/ Bordon.2016.37925

Ávila Ruiz, Rosa María y Duarte Piña, Olga (2017). Las unidades didácticas como eje de integración del conocimiento práctico profesional en la educación patrimonial. Cambios epistemológicos del profesorado en formación. En Ramón Martínez Medina, Roberto García-Morís y Carmen Rosa García Ruiz (Eds.), Investigación en didáctica de las ciencias sociales. Retos, preguntas y límites de investigación (pp. 25-33). Córdoba: Universidad de Córdoba.

Ballesteros Valladares, Paloma, Domingo Fominaya, María, Fontal Merillas, Olaia y Cirujano Gutiérrez, Concepción (2013). Plan Nacional de Educación y Patrimonio. Madrid: Ministerio de Educación, Cultura y Deporte.

Calaf Masachs, Roser, San Fabián Maroto, José Luis y Gutiérrez Berciano, Sué (2017). Evaluación de programas educativos en museos. Una nueva perspectiva. Bordón, 69 (1), 45-65. https://doi.org/10.13042/Bordon.2016.42686

Castellón, Federico y Martínez, Rafael (2001). La difusión del patrimonio histórico en Málaga. El gabinete pedagógico de bellas artes. Difusión del Patrimonio Histórico. Revista Jábega 89, 71-82.

Concejalía de Turismo, Educación y Universidad de Lorca (2018). Conoce Lorca. Disponible en: http://www.lorcaturismo.es/situacion/situacion.asp?id=6

Cuenca López, José María, Estepa Giménez, Jesús y Martín Cáceres, Myriam José (2011). El patrimonio cultural en la educación reglada. Patrimonio cultural de España, 5, 45-58. Disponible en: http://rabida.uhu.es/dspace/bitstream/handle/10272/9437/ El_patrimonio_cultural.pdf?sequence $=2$.

Cuenca López, José María y Martín Cáceres, Myriam (2015). Educomunicación del patrimonio. Educatio Siglo XXI, 33 (1), 33-54. http://dx.doi.org/10.6018/j/222491

Escarbajal de Haro, Andrés, y Martínez de Miguel López, Silvia. (2012). El papel de la educación y los museos en la inclusión social. Una contribución desde la animación sociocultural. Educatio Siglo XXI, 30(2), 445-466. Disponible en: https://revistas. um.es/educatio/article/view/160921.

Escribano Miralles, Ainoa y Molina Puche, Sebastián (2015a). La presencia de la historia del arte en el nuevo curriculum de educación primaria. Iber, 79, 15-24.

Escribano Miralles, Ainoa y Molina Puche, Sebastián (2015b). La importancia de salidas escolares y museos en la enseñanza de las ciencias sociales en educación infantil. 
Revista de Humanidades, 38 (2019). p.11-36. ISSN 1130-5029

Análisis de un caso a partir del modelo CIPP. Clío, 41. Disponible en: http://clio. rediris.es/n41/articulos/EscribanoMolina2015.pdf.

Estepa Giménez, Jesús (2013). La educación patrimonial en la escuela y el museo. Investigación y Experiencias. Huelva: Universidad de Huelva.

Fontal Merillas, Olaia (2003). La educación patrimonial. Teoría y práctica en el aula, el museo e Internet. Gijón: Ediciones Trea SL.

Fontal Merillas, Olaia (2013). La educación patrimonial: del patrimonio a las personas. Gijón: Ediciones Trea SL.

Fontal Merillas, Olaia e Ibáñez Etxeberría, Alex (2015). Estrategias e instrumentos para la educación patrimonial en España. Educatio Siglo XXI, 33 (1), 15-32. http:// dx.doi.org/10.6018/j/222481.

Fontal Merillas, Olaia, Ibáñez Etxeberria, Alex, Martínez Rodríguez, Marta y Rivero Gracia, Pilar. (2017). El patrimonio como contenido en la etapa de Primaria. Del currículum a la formación de maestros. Revista Electrónica Interuniversitaria de Formación del Profesorado, 20(2), 79-95. http://dx.doi.org/10.6018/ reifop.20.1.286321.

Fontal Merillas, Olaia y Marín Cepeda, Sofía (2018). Nudos Patrimoniales. Análisis de los vínculos de las personas con el patrimonio personal. Arte, Individuo y Sociedad 30 (3), 483-500. http://dx.doi.org/10.5209/ARIS.57754.

Fontal Merillas, Olaia, Marín-Cepeda, Sofía, García-Ceballos, Silvia, Martínez Rodríguez Marta y Sánchez Macías Inmaculada (2018). Miradas al patrimonio. Nueve años del OEPE. En Esther López Torres, Carmen Rosa García Ruiz y María Sánchez Agustí (Edts.), Buscando formas de enseñar. Investigar para innovar en didáctica de las Ciencias Sociales (pp. 585-596). Valladolid: Ediciones Universidad de Valladolid.

García Sampedro, Marta y Gutiérrez Berciano, Sue. (2018). El museo como espacio multicultural y de aprendizaje: algunas experiencias inclusivas. Revista Anual de Historia del Arte, 24, 117-128. Disponible en https://dialnet.unirioja.es/descarga/ articulo/6516441.pdf

González-Monfort, Neus (2011) La presencia del patrimonio cultural en los currícula de educación infantil, primaria y secundaria obligatoria en España. Patrimonio cultural de España, 5, 58-75. http://dx.doi.org/10.6018/j/222521.

González-Monfort, Neus y Magoga, Alessandro (2014). Los NNATS y sus representaciones sociales sobre el patrimonio cultural. La experiencia de Cajamarca (Perú). En Olaia Fontal Merillas, Alex Ibáñez-Etxeberria y Lorenzo Martín Sánchez, (Eds.), Reflexionar desde las experiencias. Una visión complementaria entre España, Francia y Brasil. Actas del II Congreso Internacional de Educación Patrimonial (pp. 925-935). Madrid: IPCE/OEPE.

Guisasola Aranzabal, Jenaro y Morentin Pascual, Maite (2007). ¿Qué papel tienen las visitas escolares a los museos de ciencias en el aprendizaje de las ciencias? Una revisión de las investigaciones. Investigación didáctica, 25 (3), 401-414. 
Estudio sobre el patrimonio de la ciudad de Lorca... - S. Martínez de Miguel y otros

Disponible en: https://ddd.uab.cat/pub/edlc/02124521v25n3/02124521v25n3p401. pdf.

Ibáñez Etxeberría, Alex, Fontal Merillas, Olaia y Cuenca López, José María, (2015). Actualidad y tendencias en Educación Patrimonial. Educatio Siglo XXI, 33 (1), 1114. http://dx.doi.org/10.6018/j/222491.

Izquierdo Peraila, Isabel, López Ruiz, Clara. y Prados Torreira, Lourdes (2014). Infancia, museología y arqueología. Reflexiones en torno a los museos arqueológicos y el público infantil. Archivo de prehistoria levantina, 30, 401-418.

Jagielska-Burduk, Alicja \& Stec, Piotr (2019). Council of Europe Cultural Heritage and Education Policy. Preserving identity and searching for a Common Core? Revista Electrónica Interuniversitaria de Formación del Profesorado, 22(1), 1-12. http:// dx.doi.org/10.6018/reifop.22.1.354641

Latorre Madueño, Gloria y Mérida Serrano, Rosario (2019). La ciudad como contexto educativo: Desarrollo de la competencia cívica en las aulas infantiles y en el alumnado con necesidades de apoyo educativo. Foro de Educación, 17(27), 203227. http://dx.doi.org/10.14516/fde.649

Ley Orgánica 2/2006, de 3 de mayo, de Educación. BOE, 4 de mayo de 2006, 106, 17158-17207. Disponible en: https:/www.boe.es/boe/dias/2006/05/04/pdfs/ A17158-17207.pdf.

Ley Orgánica 8/2013, de 9 de diciembre, para la mejora de la calidad educativa. $B O E, 10$ de diciembre de 2013, 295, 97858-97921. Disponible en: http://www.boe.es/boe/ dias/2013/12/10/pdfs/BOE-A-2013-12886.pdf.

López Cruz, I. (2014). La Educación Patrimonial. Análisis del tratamiento didáctico del patrimonio en los libros de texto de CCSS en la Enseñanza Secundaria (Tesis doctoral). Universidad de Huelva, Huelva.

Martínez Gil, Tania (2014). El patrimonio religioso y la educación patrimonial. Un camino por recorrer. En Olaia Fontal Merillas, Alex Ibáñez-Etxeberría y Lorenzo Martín Sánchez, Lorenzo. (Eds.), Reflexionar desde las experiencias. Una visión complementaria entre España, Francia y Brasil. Actas del II Congreso Internacional de Educación Patrimonial (pp.1185-1198). Madrid: IPCE/OEPE.

Méndez Andrés, Ramón (2017). Vincular la visita escolar al museo con la realidad del aula. Intereses y prácticas de los docentes que visitan el museo del ferrocarril de Madrid. En Ramón Martínez Medina, Roberto García-Morís y Carmen Rosa García Ruiz (Eds.), Investigación en didáctica de las ciencias sociales. Retos, preguntas y límites de investigación (pp. 237-248). Córdoba: Universidad de Córdoba.

Ministerio de Cultura (2011). Conociendo a nuestros visitantes. Estudio de público en museos del Ministerio de Cultura. Laboratorio Permanente de público de museos. Madrid: Subdirección General de Museos Estatales.

Ministerio de Educación (2015). Encuesta de hábitos y prácticas culturales en España 2014-2015. Madrid: Subdirección General de Documentación y Publicaciones. 
Revista de Humanidades, 38 (2019). p.11-36. ISSN 1130-5029

Molina Puche, Sebastian y Pinto, Helena. (2015). La educación patrimonial en los currículos de ciencias sociales en España y Portugal. Educatio Siglo XXI, 33, 1, 103-128. http://dx.doi.org/10.6018/j/222521

Monteagudo López Menchero, Jesús. (2014). El concepto de Patrimonio. Un enfoque plural a diversas escalas. En Gregorio Canales Martínez, José Manuel Pérez Burgos y Felio Lozano Quijada (Eds.), Nueva Tabarca, un desafio multidisciplinar (pp. 17-46). Alicante: Instituto Alicantino de Cultura Juan Gil-Alber. Disponible en: http://web.ua.es/es/seus/orihuela/documentos/publicaciones/2014-2015/libronueva-tabarca.pdf.

Ott, Michela \& Pozzi, Francesca. (2011). Towards a new era for Cultural Heritage Education. Discussing the role of ICT. Computers in Human Behavior, 27 (4), 1365-1371. doi: 10.1016 / j.chb.2010.07.031

Piedra Vera, Shubert Enrique (2018). Factores que aportan las actividades lúdicas en los contextos educativos. Cognosis, 4 (1), 93-108. Disponible en https://revistas.utm. edu.ec/index.php/Cognosis/article/download/1211/1403/

Pinto, Helena (2013). Usos del patrimonio en la didáctica de la Historia: Perspectivas de alumnos y profesores portugueses relativas a identidad y conciencia histórica. Educatio Siglo XXI, 31 (1), 61-87. Recuperado de: https://revistas.um.es/educatio/ article/view/175341.

Real Decreto 198/2014. Currículo de la educación Primaria en la Comunidad Autónoma de la Región de Murcia. (BORM, 6 de septiembre de 2014).

Real Decreto 1513/2006. Enseñanzas mínimas de Educación Primaria (BOE, 8 de diciembre de 2006).

Reyes Leoz, José Luis de los (2016). Museos y centros escolares. Entornos de aprendizaje. Unes, 1, 80-96.

UNESCO (2011). ¿Qué es el Patrimonio Cultural Inmaterial? Recuperado de: http:// www.unesco.org/culture/ich/doc/src/01851-ES.pdf 